\title{
LOCALLY EQUATIONAL COMPLETENESS OF RINGS AND SEMIGROUPS
}

\author{
M. R. CHIARO
}

\begin{abstract}
Hu has developed locally equational classes as a generalization of equational classes. It is shown here that the lattice of equational classes of rings is a proper sublattice of the lattice of locally equational classes of rings but the locally equationally complete rings are precisely the equationally complete rings. Using the equational and locally equational class operators the locally equationally complete classes of semigroups are shown to be those that are equationally complete.
\end{abstract}

1. Introduction. In [3], Hu defined locally equational classes and established that the collection of locally equational classes of universal algebras of a fixed type forms a lattice which includes the lattice of equational classes as a sublattice.

Tarski [8] has shown the equationally complete rings to be the $p$-rings and $p$-zero rings. Contained in this paper is an example demonstrating that the lattice of equational classes of rings is a proper sublattice of the lattice of locally equational classes of rings, prompting investigation of the possible existence of rings $\Re$ that are locally equationally complete but $\mathbf{E}(\Re)$ is not an atom in the lattice of locally equational classes of rings or is not an atom in the lattice of equational classes of rings. The locally equationally complete rings are shown to be precisely the equationally complete rings.

Kalicki and Scott [5] have characterized the equationally complete classes of semigroups which are shown here to be the locally equationally complete classes of semigroups. The proof techniques are operator-theoretic in nature and are more elegant than the equational class case as the notion of identities is avoided.

Basic concepts can be found in [1] and [2].

\section{Preliminaries.}

Definition. Let $\mathscr{K}$ be a class of algebras of the same type. Denote by $\mathbf{E}_{L}(\mathscr{K})$ the class of all algebras $\mathfrak{U}=\langle A ; F\rangle$ having the following property: for each finite subset $U$ of $A$, there exists a finite family $\left\{\mathfrak{B}_{i}\right\}_{i \in I}$ in $\mathscr{K}$ and, for each $i \in I$, a finite subset $V_{i}$ of $B_{i}$ such that every identity valid in $V_{i}$ for all $i \in I$ is also valid in $U$. For a single algebra $\mathscr{B}$ denote $\mathbf{E}_{L}(\{\mathscr{B}\})$ by $\mathbf{E}_{L}(\mathscr{B})$.

Definition. Let $\mathcal{K}$ be a class of algebras of the same type. $\mathcal{K}$ is locally equational if $\mathbf{E}_{L}(\mathcal{K})=\mathscr{K} . \mathbf{E}_{L}(\mathcal{K})$ is the locally equational class generated by

Received by the editors October 22, 1975 and, in revised form, June 16, 1976.

AMS (MOS) subject classifications (1970). Primary 08A15; Secondary 08A25. 
$\mathcal{K} . \mathbf{E}(\mathcal{K})$ denotes the equational class generated by $\mathcal{K}$.

Definition. If $\mathfrak{A}=\langle A ; F\rangle$ is an algebra, a local system of subalgebras of $\mathfrak{A}$ is a set of subalgebras of $\mathfrak{A}$ which is directed by set-inclusion and whose set-theoretic union is $A$. If $\mathcal{L}$ is a nonempty set of algebras such that any two members of $\mathcal{L}$ are subalgebras of some third member of $\mathcal{L}$, there is a unique algebra having $\mathcal{L}$ as a local system of subalgebras and this algebra is called the directed union of $\mathcal{L}$.

Definition. Let $\mathbf{D}, \mathbf{H}, \mathbf{S}, \mathbf{P}_{\boldsymbol{F}}$ and $\mathbf{P}$ denote the operators of forming, respectively, directed unions, homomorphic images, subalgebras, direct products of finite families and direct products of arbitrary families.

THEOREM 2.1 [3]. If $\mathcal{K}$ is a class of algebras of the same type then $\mathbf{E}_{L}(\mathcal{K})=\operatorname{DHSP}_{F}(\mathcal{K})$ and $\mathbf{E}_{L}(\mathcal{K}) \subseteq \mathbf{E}(\mathcal{K})$. If $\mathcal{K}$ is a finite class of finite algebras of the same type then $\mathbf{E}_{L}(\mathscr{K})=\mathbf{E}(\mathcal{K})$.

THEOREM 2.2 [3]. For a fixed type $\tau$ of algebras, the locally equational classes of type $\tau$ form a lattice under set-inclusion which includes as a sublattice the lattice of equational classes of type $\tau$.

Definition. Let $\mathfrak{U}$ be a nontrivial algebra of type $\tau$. $\mathfrak{A}$ is equationally complete if $\mathbf{E}(\mathfrak{U})$ is an atom in the lattice of equational classes of type $\tau$. Let $\mathscr{K}$ be a class of algebras of type $\tau$. $\mathcal{K}$ is equationally complete if $\mathbf{E}(\mathcal{K})$ is an atom in the lattice of equational classes of type $\tau$.

Definition. Let $\mathfrak{A}$ be a nontrivial algebra of type $\tau$. $\mathfrak{A}$ is locally equationally complete if $\mathbf{E}_{L}(\mathfrak{U})$ is an atom in the lattice of locally equational classes of type $\tau$. Let $\mathcal{K}$ be a class of algebras of type $\tau$. $\mathcal{K}$ is locally equationally complete if $\mathbf{E}_{\boldsymbol{L}}(\mathcal{K})$ is an atom in the lattice of locally equational classes of type $\tau$.

Definition. For a prime number $p$, a commutative ring $\Re$ is called a $p$-ring if $p a=0$ and $a^{p}=a$ for each element $a$. $\Re$ is called a $p$-zero-ring if $p a=0=a \cdot b$ for all elements $a$ and $b$.

DEFINITION. For any positive integer $n>1, \Re_{n}$ denotes the ring $\left\langle Z_{n}\right.$; $0,-,+, \cdot>$ where $Z_{n}$ is the set of all nonnegative integers less than $n$, + and - are the binary operations of addition and multiplication modulo $n,-$ is the unary operation of additive inverse and 0 is the nullary operation of additive identity. $3 \Re_{n}$ denotes the same ring as $\Re_{n}$ except $\cdot$ is defined so that $a \cdot b=0$ for all elements $a$ and $b$.

THEOREM 2.3 [7]. Let $\Re$ be a nontrivial p-ring. $\Re$ is isomorphic to a subdirect power of $\Re_{p}$.

THEOREM 2.4 [8]. Every ring $\Re$ with more than one element has a subring which is homomorphic, for some prime number $p>1$, either to $\Re_{p}$ or $3 \Re_{p}$.

3. Example. The following example shows that the locally equational class generated by a ring $\Re$ need not be an equational class. Hence the lattice of equational classes of rings is a proper sublattice of the lattice of locally equational classes of rings and atoms of the latter are of interest. 
Let $\Re=\langle R ; 1,-,+, \cdot\rangle$ be the following ring: $R$ is the multiplicative group of complex roots of unity, + is the usual field multiplication, - is the unary operation of forming reciprocals, 1 is the nullary operation corresponding to the multiplicative identity of the complex field and - is the trivial multiplication where $r \cdot s=1$ for all $r, s \in R$.

All members of DHSP $_{F}(\Re)$ consist of elements of finite (group) order. But the direct power $\Re^{\omega}$ contains an element of infinite order so that $\mathfrak{R}^{\omega} \notin \mathbf{E}_{L}(\mathfrak{R})$. Hence $\mathbf{E}_{L}(\Re)$ is not an equational class and is properly contained in $\mathbf{E}(\Re)$.

\section{Locally equationally complete rings.}

LEMMA 4.1. The ring $\Re_{p}$ is locally equationally complete for any prime number $p>1$.

Proof. Let $\mathscr{E}_{L}$ be a nontrivial locally equational class of rings contained in $\mathbf{E}_{L}\left(\Re_{p}\right)$. Let $\Re \in \mathcal{E}_{L}$ be nontrivial. $\Re \in \mathbf{E}_{L}\left(\Re_{p}\right)=\mathbf{H S P}\left(\Re_{p}\right)$ by Theorem 2.1 so $\Re$ is isomorphic to $\Re^{\prime} / \Im$ where $\Re^{\prime} \in \mathbf{S P}\left(\Re_{p}\right)$ and $\mathfrak{\Im}$ is an ideal of $\Re^{\prime}$.

Since $\Re^{\prime}$ is a subring of $\Pi_{\lambda \in \Lambda} \Re_{\lambda}$ for some $\Lambda$ where each $\Re_{\lambda}$ is $\Re_{p}$, there exists $\lambda_{0} \in \Lambda$ such that $\left\{f\left(\lambda_{0}\right) \mid f \in \mathbf{R}^{\prime}\right\}=Z_{p}$. If $U=\left\{u_{1}, \ldots, u_{m}\right\} \subseteq Z_{p}$, let $f_{1}, \ldots, f_{m} \in R^{\prime}$ such that $f_{i}\left(\lambda_{0}\right)=u_{i}, i=1, \ldots, m$. Then if $\left\{f_{i}+\Im\right) i=$ $1, \ldots, m\}=V$, since identities in $V$ must hold componentwise, each identity of $V$ is an identity of $U$. Therefore $\Re_{p} \in \mathbf{E}_{L}\left(\Re^{\prime} / \mathfrak{S}\right)$ and $\Re_{p} \in \mathbf{E}_{L}(\Re)$. Hence $\mathbf{E}_{L}\left(\Re_{p}\right)=\mathscr{E}_{L}$ and $\Re_{p}$ is locally equationally complete.

LEMMA 4.2. Every nontrivial p-ring is locally equationally complete.

Proof. Any nontrivial $p$-ring is isomorphic to a subalgebra of a direct power of $\Re_{p}$ by Theorem 2.3. Hence $\Re \in \mathbf{H S P}\left(\Re_{p}\right)=\mathbf{E}\left(\Re_{p}\right)=\mathbf{E}_{L}\left(\Re_{p}\right)$. Since $\mathbf{E}_{L}(\Re) \subset \mathbf{E}_{L}\left(\Re_{p}\right)$ and $\Re_{p}$ is locally equationally complete, $\mathbf{E}_{L}(\Re)=\mathbf{E}_{L}\left(\Re_{p}\right)$.

COROLlaRY 4.3. For any p-ring $\Re, \mathbf{E}_{L}(\Re)=\mathbf{E}(\Re)$.

Proof. $\mathbf{E}_{L}(\Re) \subseteq \mathbf{E}(\Re)$ by Theorem 2.1. $\Re \in \mathbf{E}\left(\Re_{p}\right)$ by Theorem 2.3 so that $\mathbf{E}(\Re) \subseteq \mathbf{E}\left(\Re_{p}\right)$. Hence $\mathbf{E}_{L}(\Re) \subseteq \mathbf{E}(\Re) \subseteq \mathbf{E}\left(\Re_{p}\right)=\mathbf{E}_{L}\left(\Re_{p}\right)$.

But $\mathbf{E}_{L}\left(\Re_{p}\right)$ is an atom by Lemma 4.2 , hence $\mathbf{E}_{L}(\Re)=\mathbf{E}_{L}\left(\Re_{p}\right)$.

The following three are analogous to the above.

LEMMA 4.4. The ring $3 \Re_{p}$ is locally equationally complete for any prime number $p>1$.

LEMMA 4.5. Every nontrivial p-zero-ring is locally equationally complete.

LEMMA 4.6. For every p-zero-ring $\Re, \mathbf{E}_{L}(\Re)=\mathbf{E}(\Re)$.

LEMMA 4.7. Every locally equationally complete ring is either a p-ring or a p-zero-ring.

PRoof. Let the ring $\Re$ be locally equationally complete. By Theorem $2.4 \Re$ has a subring which is homomorphic, for some prime number $p>1$, either to $\Re_{p}$ or $3 \Re_{p}$. By Lemma 4.1 (Lemma 4.4), $\mathbf{E}_{L}\left(\Re_{p}\right)=\mathbf{E}_{L}(\Re)$ and $\Re \in \mathbf{E}_{L}\left(\Re_{p}\right)$ $\left(\mathbf{E}_{L}\left(3 \Re_{p}\right)=\mathbf{E}_{L}(\Re)\right.$ and $\left.\Re \in \mathbf{E}_{L}\left(3 \Re_{p}\right)\right)$. 
But among the identities of any subset of $\Re_{p}\left(3 \Re_{p}\right)$ are $p a=0$ and $a^{p}=a$ $(p a=0=a \cdot b)$. Hence $\Re$ is either a $p$-ring or a $p$-zero-ring.

5. Locally equational completeness of semigroups. Let $A S$ be the class of all semigroups.

1. $S L$ is the class of all semilattices, that is all algebras in $A S$ where $x+y=y+x$ and $x+x=x$ hold.

2. $L F$ is the class of all left algebras, that is all algebras in $A S$ where $x+y=x$ holds.

3. $R G$ is the class of all right algebras, that is all algebras in $A S$ where $y+x=x$ holds.

4. $C S$ is the class of all constant algebras, all algebras in $A S$ where $x+y=z+w$ holds.

5. If $p \geqslant 2$ is a prime, then the class $p$-GR of $p$-groups is all algebras in $A S$ for which both $x+y=y+x$ and $p x+y=y$ hold.

Let $C M=\{S L, L F, R G, C S, p-G R\}$.

For $K \in C M, \mathcal{C}(K)$ is the characteristic algebra defined as follows:

1. $\mathcal{C}(S L)=\left\langle\{0,1\},+_{S L}\right\rangle$ where $+_{s L}$ is defined by

\begin{tabular}{c|cc}
$+_{S L}$ & 0 & 1 \\
\hline 0 & 0 & 0 \\
1 & 0 & 1
\end{tabular}

2. $\mathcal{C}(L F)=\left\langle\{0,1\},+_{L F}\right\rangle$ where $+_{L F}$ is defined by

\begin{tabular}{c|cc}
$+_{L E}$ & 0 & 1 \\
\hline 0 & 0 & 0 \\
1 & 1 & 1
\end{tabular}

3. $\mathcal{C}(R G)=\left\langle\{0,1\},+_{R G}\right\rangle$ where $+_{R G}$ is defined by

\begin{tabular}{c|cc}
$+_{R G}$ & 0 & 1 \\
\hline 0 & 0 & 1 \\
1 & 0 & 1
\end{tabular}

4. $\mathcal{C}(C S)=\left\langle\{0,1\},+_{C S}\right\rangle$ where $+_{c S}$ is defined by

\begin{tabular}{c|cc}
$+_{C S}$ & 0 & 1 \\
\hline 0 & 0 & 0 \\
1 & 0 & 0
\end{tabular}

5. If $p$ is a prime then $\mathcal{C}(p-G R)=\left\langle\{0,1, \ldots, p-1\},+_{p-G R}\right\rangle$ where $+_{p-G R}$ is the operation of addition $\bmod p$.

Theorem 5.1 [5]. If $K \in C M$ then $\mathbf{E}(\mathcal{C}(K))=K$.

TheOREM 5.2 [5]. If $K \in C M$ and $\mathfrak{U} \in K \ni|A| \geqslant 2$, then $\mathcal{C}(K)$ is isomorphic to a subalgebra of $\mathfrak{A}$.

Theorem 5.3 [5]. If $\mathfrak{A} \in A S \ni|A| \geqslant 2$, then for some $K \in C M, \mathcal{C}(K)$ is a homomorphic image of a subalgebra of $\mathfrak{A}$. 
THEOREM 5.4. If $\mathfrak{A}$ is a semigroup and is locally equationally complete then there exists $K \in C M$ such that $\mathfrak{A} \in K$.

Proof. By Theorem 5.3, for some $K \in C M \mathcal{C}(K)$ is an element of $\mathbf{E}_{L}(\mathfrak{A})$. But $|\mathcal{C}(K)|<\infty, \mathbf{E}_{L}(\mathcal{C}(K))=\mathbf{E}(\mathcal{C}(K))$ and $\mathbf{E}(\mathcal{C}(K))=K$. Hence $\mathbf{E}_{L}(\mathfrak{A})=$ $K$ and $\mathfrak{A} \in K$.

THEOREM 5.5. All classes in CM are locally equationally complete.

Proof. Assume $K \in C M$ is not locally equationally complete and let $K^{\prime} \subset K$ where $K^{\prime}$ contains an algebra $\mathfrak{A} \ni|A|>1$. Since $\mathfrak{A} \in K^{\prime} \subset K, \mathfrak{A} \in$ $K$ and $\mathcal{C}(K) \in \mathbf{E}_{L}(\mathfrak{A})$ by Theorem 5.2. But $\mathbf{E}_{L}(K)=\mathbf{E}_{L}(\mathcal{C}(K)) \subseteq \mathbf{E}_{L}(\mathfrak{U}) \subseteq$ $\mathbf{E}_{L}\left(K^{\prime}\right)$ so that $\mathbf{E}_{L}(K) \subseteq \mathbf{E}_{L}\left(K^{\prime}\right)$ and $K$ is indeed locally equationally complete.

THEOREM 5.6. If $K^{\prime}$ is a locally equationally complete class of semigroups then $K^{\prime}$ is an element of $C M$.

Proof. Let $\mathfrak{A} \in K^{\prime}$ where $|A|>1$. Since $K^{\prime}$ is an atom, $\mathfrak{A}$ is locally equationally complete so by Theorem 5.4 there exists $K \in C M \ni \mathfrak{A} \in K$. But $K$ is an atom by Theorem 5.5 so that $K=\mathbf{E}_{L}(\mathfrak{Q})=K^{\prime}$ and $K^{\prime} \in C M$.

\section{REFERENCES}

1. P. M. Cohn, Universal algebra, Harper and Row, New York, 1965.

2. G. Gratzer, Universal algebra, Van Nostrand, Princeton, N. J., 1968.

3. T. K. Hu, Locally equational classes of universal algebras, Chinese J. Math. 1 (1973), 143-165.

4. On the topological duality for primal algebra theory, Algebra Universalis 1 (1971), $152-154$.

5. J. Kalicki and D. Scott, Equational completeness of abstract algebras, Nederl. Akad. Wetensch. Proc. Ser. A 58 (1955), 650-659.

6. N. H. McCoy, Rings and ideals, Carus Monograph No. 8, Math. Assoc. of America, 1948.

7. N. H. McCoy and D. Montgomery, A representation of generalized Boolean rings, Duke Math. J. 3 (1937), 455-459.

8. A. Tarski, Equationally complete rings and relation algebras, Indag. Math. 18 (1956), 39-46.

Department of Mathematics, Claremont Graduate School, Claremont, California 91711

Current address: 708 N. 13, Pocatello, Idaho 83201 